\title{
Propuesta de ambientes de aprendizaje para la promoción de la modelación matemática desde la perspectiva crítica ${ }^{1}$
}

\author{
Proposal learning environments for promoting \\ mathematical modeling critical perspective
}

\section{Proposta ambientes de aprendizagem para promover a modelagem matemática perspectiva crítica}

Recibido: mayo de 2013

Aceptado: agosto de 2013
Angela Maritza Bustos Motavita ${ }^{2}$

Geraldine Bustos Motavita ${ }^{3}$

Yenny Carolina Novoa Parra ${ }^{4}$

\section{Resumen}

Este documento reporta algunas reflexiones de una investigación ${ }^{5}$ de carácter cualitativo, que hizo uso de la metodología investigación-acción $y$, desarrollo una propuesta de enseñanza-aprendizaje para visualizar los procesos de Modelación Matemática usados por estudiantes de noveno grado de educación básica secundaria de un colegio Distrital de Bogotá al abordar Ambientes de Aprendizaje desde la propuesta de Skovsmose.

Palabras Clave: Educación Matemática Critica; Aprendizaje; Procesos Cognitivos; Modelización; Ambientes de Aprendizaje; Modelo; Función Lineal; Enseñanza; Metodología de trabajo en el aula.

\begin{abstract}
This paper reports some investigación1de reflections of a qualitative nature, which made use of action research methodology and develop a proposal for teaching and learning to visualize mathematical modeling processes used by freshmen basic secondary education of a school district Bogota to address Learning Environments from Skovsmose proposal.
\end{abstract}

Keywords: Critical Mathematics Education, Learning, Cognitive Processes, Modelling, Learning Environments; Model; Linear Function; Education; Methodology classroom work.

\section{Resumo}

Este artigo apresenta algumas reflexões investigación1de de natureza qualitativa, que fez uso da metodologia de pesquisa-ação e desenvolver uma proposta de ensino e aprendizagem para visualizar os processos de modelagem

1 Artículo de Investigación.

2 Universidad Distrital Francisco Jose de Caldas. Bogotá, Colombia. Contacto: angelmar.bm@hotmail.com

3 Universidad Distrital Francisco Jose de Caldas. Bogotá, Colombia. Contacto: babylu1102@hotmail.com

4 Universidad Distrital Francisco Jose de Caldas. Bogotá, Colombia. Contacto: ycnovoap@correo.udistrital.edu.co

5 Para profundizar, consultar, Bustos, A. (2012) 
matemática utilizadas pelos calouros do ensino secundário básico de um distrito escolar Bogotá para enfrentar ambientes de aprendizagem da proposta Skovsmose.

Palavras-chave: Educação Matemática Crítica, Aprendizagem, Processos Cognitivos, Modelagem, Ambientes de Aprendizagem; modelo; Função Linear, Educação, Metodologia de trabalho em sala de aula.

\section{Presentación del problema}

De acuerdo a lo planteado en los Lineamientos Curriculares (MEN, 1998), es necesario involucrar los contenidos matemáticos con la realidad de los estudiantes para dar sentido a la actividad matemática hecha en el aula. Sin embargo, desde las prácticas docentes ${ }^{6}$ hechas por Bustos (2013), este planteamiento no se evidenciaba en el aula de clase. Así como también lo evidencia Villa (2009) con el caso de Alberto, quien considera que "las situaciones reales abordadas en el aula de clase, deberían ir más allá de una re-vestidura de conceptos en un contexto artificial" En este sentido, la Modelación Matemática desde la perspectiva crítica, permite vincular el contexto socio-cultural en el cual están inmersos los estudiantes con la matemática, lo cual, les permite como lo menciona Valero (2002) el desarrollo de competencias democráticas necesarias para ser actores responsables en su sociedad.

En este sentido, se considera pertinente preguntar ¿Cómo usan los procesos de Modelación Matemática los estudiantes al abordar Ambientes de Aprendizaje desde la Educación Matemática Crítica?

\section{Marco teórico}

Inicialmente, para esta propuesta se tiene en cuenta la Educación Matemática desde la perspectiva crítica, debido a que en el aula se eliminan las relaciones de poder y el estudiante hace parte activa en su proceso de enseñanza-aprendizaje, haciendo que este proceso como menciona Jacobini (2004) sean vistos los posicionamientos críticos y las posturas democráticas, que luego serán útiles para que el estudiante transforme su sociedad. De modo que, además de adquirir el conocimiento, el estudiante adquiere las habilidades necesarias para usar estos conocimientos es decir ofrece la oportunidad según Valero (1996) de que se represente, analice, modele y tome decisiones respecto a una situación de su cotidianidad.

En este sentido, los Ambientes de Aprendizaje propuestos por Skovsmose (2000) permiten visualizar las diversas formas en que se trabaja en el aula, al combinar las formas de organizar la actividad de los estudiantes con el tipo de referencia, ambientes que para el enfoque de la Educación Matemática Crítica, van desde lo considerado como "tradicional", hasta el Ambiente tipo 6 considerado como "ideal" como se muestra en la tabla.

Tabla 1

\begin{tabular}{|c|c|c|c|}
\hline & & \multicolumn{2}{|c|}{$\begin{array}{l}\text { Formas de organización de la } \\
\text { actividad de los estudiantes }\end{array}$} \\
\hline & & $\begin{array}{c}\text { Paradigma del } \\
\text { ejercicio }\end{array}$ & $\begin{array}{l}\text { Escenarios de } \\
\text { investigación }\end{array}$ \\
\hline \multirow{3}{*}{$\begin{array}{l}\text { Tipo de } \\
\text { referencia }\end{array}$} & $\begin{array}{l}\text { Matemáticas } \\
\text { puras }\end{array}$ & (1) & (2) \\
\hline & Semirrealidad & (3) & (4) \\
\hline & $\begin{array}{l}\text { Situaciones de la } \\
\text { vida real }\end{array}$ & (5) & (6) \\
\hline
\end{tabular}

Fuente: Elaboración propia

Explícitamente, el ambiente tipo (6) permite sacar de la realidad del estudiante la situación, de modo que éste le dé sentido a la actividad matemática que está realizando, en ella el estudiante tiene que explorar, comprender y modelar, además de llegar a propuestas de solución de la misma tanto matemáticas como sociales de ser necesario.

Finalmente se tiene en cuenta la Modelación Matemática como lo identifica Caldeira (2007) citado en Araùjo (2009. p.60), como "un proceso de obtención y validación de un conjunto de

símbolos y relaciones matemáticas presentes en un objeto estudiado" ". Dicho conjunto de símbolos y

6 Las practicas docentes en la Licenciatura con Educación Básica con Énfasis en Matemáticas (LEBEM) de la Universidad Distrital Francisco José de Caldas, se realizan desde el momento en que el estudiante para profesor inicia su cuarto semestre y finalizan en el noveno semestre de la carrera; dichas prácticas se llevan a cabo en diferentes colegios distritales de la ciudad de Bogotá.

7 Traducción realizada para efectos del texto. 
relaciones matemáticas, se considera como el modelo matemático pues, este como lo menciona Biembengut \& Hein, [s.f.], permite vincular la realidad con la matemática, y es la síntesis de la comprensión de la situación dada por parte del resolutor de la problemática en cuestión.

Éste proceso de obtención del modelo, parte de los intereses de los estudiantes e identificación de las problemáticas de su entorno, para luego dar paso a la exploración, sistematización y análisis de resultados, validando el modelo matemático y proponiendo distintas soluciones a la problemática planteada.

\section{Metodología}

Siguiendo el marco de la investigación-acción y la propuesta de Skovsmose para generar un Ambiente de Aprendizaje tipo 6, la primera fase consistió en el reconocimiento de las problemáticas sociales de la población ${ }^{8}$ usando como método la cartografía social. En el cual, los estudiantes realizaban el mapa de los alrededores de su colegio, identificando los lugares más representativos positiva y negativamente, seguido de la dramatización de un día en la cotidianidad de cada uno de ellos. A partir de lo cual, se identificaron como problemáticas sociales presentes en la realidad de los estudiantes, la drogadicción, los embarazos adolescentes, el pandillismo y la inseguridad.

Como segunda fase, se propuso generar una discusión que permitiera reconocer los posituras de los estudiantes respecto a las problemáticas planteadas inicialmente, por medio de la temática "deserción escolar", teniendo como eje la pregunta ¿Qué consecuencias conlleva que un estudiante deje de asistir a clases? Y Finalmente en la tercera fase se encuentra el Ambiente tipo6, debido a que los estudiantes escogen sus problemáticas y trabajan a partir de ellas para posteriormente tratar de elaborar un modelo matemático por medio de la denominada Modelación Matemática. De acuerdo a: 1. Elección del tema: cada grupo de estudiantes elige el tema de su interés de acuerdo a lo expuesto por el docente; 2. Familiarización con el tema a modelar:
Experimentación e investigación de los distintos tópicos de la situación; 3. Delimitación del problema y formulación: Elige uno de los tópicos investigados para profundizar; 4.Elaboración del modelo matemático: Experimentación y validación de comportamientos y características del problema elegido de modo que pueda ser modelado; 5.Organización del trabajo escrito y exposición oral: Presentación de los resultados obtenidos mediante la investigación hecha del tema.

Se realizó el análisis cualitativo de la información, partiendo de los videos tomados, los trabajos hechos por los estudiantes y el diario de campo llevado por el docente, en el cual se tiene en cuenta la selección de la información que hacen los estudiantes, la pertinencia de la información, el paso del lenguaje matemático al lenguaje natural y viceversa, así como la necesidad de verificar que el modelo creado, finalizando con las conclusiones y soluciones parciales al problema.

Observando en la primera categoría que, aunque los estudiantes intentaron la recolección de información por medio de encuestas que diseñaban, no se le dio importancia a los datos recolectados, debido a que posteriormente se remitían a sitios web que les permitía conocer las opiniones de otras personas sobre el tema indagado, a pesar de ello, los estudiantes organizaron la información en diagramas de árbol, ya que allí relacionaban las causas y consecuencias de la problemática trabajada. A partir de lo cual, los estudiantes identificaban además de la forma cómo organizar lo observado en las lecturas, las relaciones que se establecen entre distintas problemáticas y algunos datos estadísticos de la cantidad de estudiantes ó jóvenes que caen en la deserción escolar, drogadicción, embarazos, entre otras problemáticas.

De esta manera se reconocieron variables cantidad-año, a partir de las cuales hacían una proyección a futuro usando como medio, la tabla de valores y la ubicación de los puntos en las graficas cartesianas como se muestra en la imagen y la transcripción. Lo cual hace parte de la segunda categoría.

8 Los Ambientes de Aprendizaje propuestos, fueron diseñados e implementados en el segundo semestre del año 2011, con un grupo de 40 estudiantes que oscilaban entre los 14 y 17 años, los cuales se encontraban cursando noveno grado de educación básica secundaria en el Colegio Distrital Paulo Freire, ubicado en la localidad de Usme en Bogotá. 
Finalmente en la tercera categoría se evidencia la no codificación de la información y las regularidades identificadas en la segunda categoría, es decir que aunque los estudiantes conocían el comportamiento general de sus datos, no realizaban un modelo, por lo cual la última categoría en la cual se debía verificar el modelo no era usada.

\section{Conclusiones}

Respecto a la implementación de los Ambientes de Aprendizaje y la Modelación matemática, se evidencio que los Ambientes 1 al 5 desarrollados en la primera fase de la investigación, dotaron de herramientas a los estudiantes para afrontar el Ambiente tipo 6. Sin embargo en el Ambiente 6 los estudiantes no construyeron un modelo matemático por lo cual no fue importante hacer su validación, lo cual dejo atrás las inferencias, interpretaciones, conclusiones y aportes a la solución a cada una de sus problemáticas, caracterizando así subjetivamente la solución del problema.

Cabe resaltar que es importante conocer el grupo de estudiantes con el cual se va a trabajar, pues, muchas veces las impresiones iníciales que se dan, no son verdaderas y lo que el docente puede caracterizar como Ambiente de Aprendizaje tipo 6, puede llegar a ser realmente tipo 4, viéndolo de este modo, hay un línea muy delgada que separa estos Ambientes de Aprendizaje, Pues, incluso aunque el estudiante escoja su problemática, esto no quiere decir que en realidad represente para él un problema. Como ejemplo de ello se muestra en la implementación del Ambiente de Aprendizaje tipo 6 un grupo de estudiantes que escogió el problema de la trata de blancas que consistía únicamente en indagar de que trataba porque era llamativo, en contraste con la estudiante que estaba preocupada por que su hermano no quería estudiar, lo cual se convertía en un problema Real que era necesario de investigar.

Para finalizar debido al tiempo y a la intención del trabajo, falto indagar por el pensamiento crítico que se forma al implementar este tipo de propuestas y el nivel que se adquiere por parte de los estudiantes en cada una de sus fases.

\section{Referencias}

Araújo, J.L. (2009). Uma Abordagem Sócio-Crítica da Modelagem Matemática: a perspectiva da educação matemática crítica. ALEXANDRIA, 2(2), 55-65.

Biembengut, M., \& Hein, N. (2006) Modelaje matemático como método de investigación en clases de matemáticas. Retrieved 11 01, 2011, from http:// www.cientec.or.cr/matematica/memoriaV.html

Biembengut, M., \& Hein, N. ([s.f.]). Modelo, Modelación, Modelaje: Métodos de enseñanza-aprendizaje de matemáticas. Retrieved 10 22, 2011, from http://matesup.utalca.cl/modelos/ articulos/modelacion_mate2.pdf

Bustos, A. (2012) Propuesta de ambientes de aprendizaje para la promoción de la modelación matemática desde la perspectiva crítica. (Tesis de grado para obtener el título de Licenciada en Educación Básica con Énfasis en Matemáticas). Universidad Distrital Francisco José de Caldas: Bogotá.

Jacobini, O. (2004). A modelagem matemática como instrumento de ação política na sala de aula. (Tesis de Doctorado). Universidade Estadual Paulista: Rio Claro.

MEN. (1998) Lineamientos Curriculares. Bogotá: MEN

Skovmose, O. (2000). Escenarios de investigación. EMA, 6 (1), 3--26.

Valero, P. (1996). La dictadura de las matemáticas: hacia una educación matemática para la paz y la democracia. In S. Bermúdez (Ed), Estrategias y experiencias para la construcción de la paz. Educación para la paz. Bogotá: Departamento de Historia- ANPAZ-Unlandes, pp. 254-268.

Valero, P. (2002). Consideraciones sobre el contexto y la educación matemática para la democracia. Quadrante. Revista Teorica e de Investigaça. 11 (1), 49-59.

Villa, J., Bustamante, C., Osorio, J., y Ocampo, D. (2009) "Sentido de Realidad y Modelación Matemática: El Caso De Alberto" Revista indução em ciência e tecnologia, 2(2), 159-180 